\title{
Minimally Invasive Sensing
}

\author{
Patricia Connolly, David Heath and Christopher McCormick \\ Bioengineering, University of Strathclyde \\ United Kingdom
}

\section{Introduction}

The key causes of mortality today include cardiovascular disease, infectious diseases, cancer and diabetes. Figure 1, from the World Health Organisation's Global Burden of Disease Report (World Health Organisation [WHO], 2006), illustrates the proportion of deaths due to the major causes. When these statistics are taken together with the age at death data as shown in Figure 2 (WHO, 2006) it can be seen that in the higher income countries, the burden of caring for the ageing population with chronic conditions will dominate healthcare needs and budgets. In the lower income countries there are still significant problems with childhood illness and infectious diseases and the challenge here is to protect the health of their younger populations.

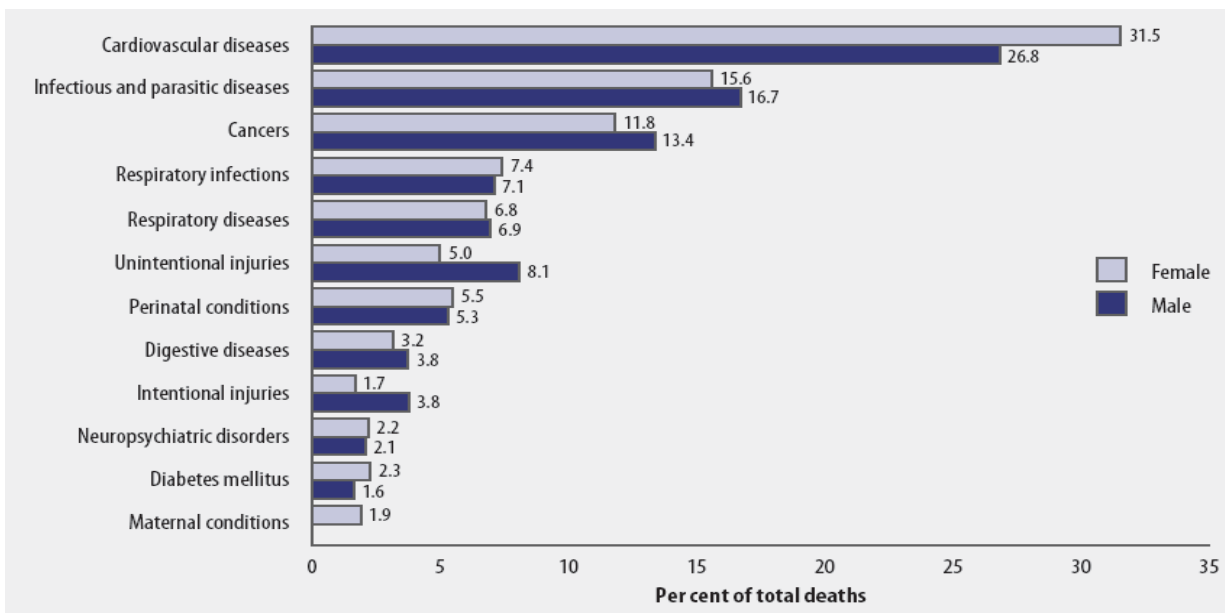

Fig. 1. Distribution of deaths by leading cause groups, male and female, worldwide, 2004 (WHO, 2006, reprinted with permission).

Whilst there are differences in the nature of the healthcare challenges between high and low-income countries, it is clear that both groups must find more effective ways of delivering healthcare into their populations at reasonable cost. This is critically important if countries are going to continue to provide effective healthcare for their citizens, whether this is privately or publicly funded. This presents challenges to pharmaceutical research, drug 
delivery, medical devices, hospital care, community care and community medicine. Chronic disease takes many people out of the community and workplace and creates an enormous and unseen group of patients requiring long term intervention and care. Secondary effects of chronic conditions generate problems in wound care, nutrition, provision of home-based medical equipment and community treatment, creating additional burdens for healthcare systems. As an example, in the UK alone the cost of chronic wounds is estimated to be $£ 2.6$ billion per annum, with 200,000 patients experiencing a chronic wound at any one time (Posnett \& Franks, 2008).

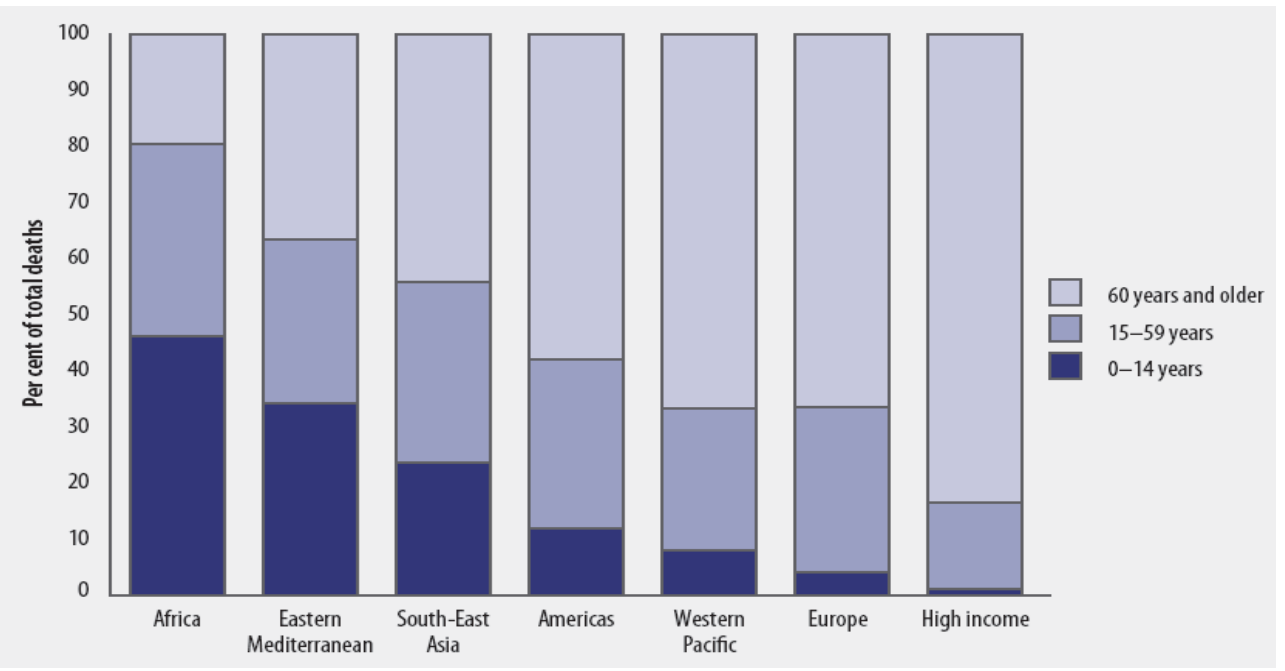

Fig. 2. Percentage deaths by age group in different global regions, 2004 (WHO, 2006, reprinted with permission)

Diagnostics and monitoring have key roles to play in optimising care, and the expectation in the biosensors community that developed in the 1980s and 1990s was that biosensors would be deployed extensively to address some of these needs. It is clear however, that despite the widespread (and frequently ingenious) development of new sensor types and technology, and the advances in device miniaturisation, there is still a notable gap between laboratory biosensing and commercially viable medical or consumer diagnostic devices. The biosensor community needs to find ways of bringing its work to the wider population for telemedicine or telehealthcare. To do this some of the fundamental problems in biosensors, which have impeded their useful deployment in healthcare, must be overcome. Some of the key challenges for practical use of clinical biosensors will now be highlighted. It is proposed that further use of minimally invasive sampling techniques for patient monitoring will allow flexibility in biosensor selection, and provide a wider range of diagnostic systems for use in the home, community or clinic.

\section{Home or frequent monitoring via wearable or minimally invasive sensors}

The field of wearable sensors that report via wireless systems is advancing, but biosensors are notably missing from current systems. Pantelopoulos \& Bourbakis, (2010) have recently 
surveyed this area and report the potential for wearable sensors for mainly ECG, EEG, blood pressure, and pO2, but glucose sensing is the only biosensor application mentioned (Pantelopoulos \& Bourbakis, 2010). Consideration of wearable sensors highlights two different clinical questions. Firstly, what are the types of parameters that would be useful to monitor, and secondly, why are there so few clinical 'on body' biosensors?

In addressing the first question, what parameters are useful for wearable sensors, there are several important factors to consider. The answer to this question requires an interdisciplinary approach. There is a question to be put to healthcare providers on what would be useful as a wearable, disposable sensor for home monitoring. Working with clinical groups, it is possible to create a profile of what would be most beneficial to their patient groups in terms of medical technology and monitoring. At present, the three leading causes of death worldwide are cancer, ischaemic heart disease, and cerebrovascular disease. It is projected that deaths attributable to these diseases will continue to rise between now and 2030, with the increase in cancer deaths being most marked (WHO, 2006). In each case, early identification of the disease has been shown to improve survival rates. High blood pressure is strongly correlated with increased risk of heart disease and stroke, and therefore technologies to enable better monitoring and early identification of these conditions may have a positive impact on reducing cardiovascular related deaths. Similarly, it has been shown in several studies that survival rates from cancer are linked to time of diagnosis. Diagnostic technologies for this purpose have been developed and are being applied in home testing kits for bowel cancer (Walsh \& Terdiman, 2003). Of great relevance to any analysis of potential parameters are the changes in lifestyle that have occurred in recent years and which are expected to continue. Most notably, obesity is an emerging problem across many developed and developing societies. It has been linked to a variety of metabolic disorders, including Type II Diabetes, cardiovascular diseases, and certain cancers. With increasingly sedentary lifestyles, it is likely to remain a major issue, and is therefore receiving considerable focus as a target for the preventative healthcare strategies increasingly being adopted. Similarly, Hospital Acquired Infections (HAI) remain an unfortunate feature across many healthcare systems, with their impact not only being felt by the affected patients, but also in driving up the treatment costs to healthcare providers. There is considerable debate about the best preventative measures to adopt to reduce HAI but any technology that proves capable of rapidly detecting such infections, or the bacteria that cause them, would be a powerful tool in such preventative strategies.

The above discussion is not comprehensive but its purpose is to provide a background to common issues facing healthcare systems across the world, and to stimulate thoughts on what parameters might usefully be measured. Looking ahead, and accepting that home monitoring is set to become a major feature of healthcare systems, what parameters could be usefully checked at home and used to adjust lifestyle or medication? As an example, if some of the key health challenges and medical conditions identified by the WHO are mapped to relevant clinical parameters, then a selection of parameters that would be useful to measure regularly and locally emerges, as shown in Table 1.

Whichever field of health is considered, a key component of any parameter analysis must be a market evaluation. The financial investment that is required to take a biosensor concept to a final product is substantial and may in itself be an explanation as to the lack of available biosensors for home settings or continuous monitoring. In this context, the question that Kissinger posed in 2005 remains key: "Do enough people want or need to have a sensor for the analyte of interest?" (Kissinger, 2005). When one considers the size of the diabetic 
market for glucose monitoring, around $\$ 7 \mathrm{bn}$ (with a small but growing segment of this given to continuous monitoring) (HSBC, 2006), it is perhaps not surprising that the glucose biosensor is the most successful of all known biosensors today, representing around $85 \%$ of the biosensors' market. In addressing the question of what type of parameter to measure the answer must clearly come from an analysis of the population base for the parameter, the clinical need, the advantages to the patient and the cost savings to be made from its proper integration in healthcare provision. This in turn will drive a true market for the sensor and ensure its uptake if properly deployed.

\begin{tabular}{|ll|}
\hline Condition & Parameter \\
Dehydration ( elderly) & electrolytes \\
Obesity ( weight loss) & ketones, tryglycerides, insulin \\
Asthma & blood parameters, compliance \\
Wound management & wound moisture, pH, bacteria \\
Diabetes & glucose, insulin, ketones \\
Cardiovascular & electrolytes, cardiac markers \\
Stress & cortisol \\
Poor nutrition & vitamins, electrolytes \\
Drug abuse & drugs of abuse \\
Drug compliance & specific sensors for medicines \\
Cancer & markers for therapy, recurrence \\
\hline
\end{tabular}

Table 1. Parameters potentially useful for home or community clinic monitoring

It is also necessary to understand the different types of markets within healthcare and alternative models of delivery within these markets. Across the world, there has been a movement towards an increasing role for Primary Health Care (WHO, 2008), with a growth in patient-centred approaches which aim to put people at the centre of their own healthcare. The practical implementation of this is causing a decentralisation of healthcare provision away from the hospital to the home, local surgeries, and pharmacies. This coincides with demands for better prevention, and early diagnosis. The driving force behind these trends is the downward pressure on the unit cost of treatment that is a major feature of today's healthcare systems.

Many of the influences identified above are well established. It is therefore pertinent to consider why such a limited number of biosensors have made an impact within this apparently favourable climate. The regulatory environment which governs such devices is an important consideration. It is beyond the scope of this chapter to detail the regulatory requirements in each region. Whatever the precise nature of the regulatory framework in any region, it is clear that it represents a significant barrier standing between a promising research result and subsequent translation into a marketable medical device product. This is certainly one explanation for the discrepancy between the volume of academic research papers reporting on biosensor development, and the rather limited number of commercially successful biosensors. Crucially, gaining regulatory approval represents a significant cost, the bulk of which is necessarily incurred at a point when the device is unable to generate sales revenue. These costs are mainly related to obtaining proof of clinical performance and generating biocompatibility or toxicology data, and to ensuring that large scale manufacture of devices is highly quality controlled. Consideration of the regulatory requirements from the outset of any medical device programme can help to minimise such costs by the correct selection of acceptable materials, and by adoption of approved design practices from the start of the process. 
This takes us to a discussion of the key biological challenges in the deployment of biosensors, either in wearable format or as implanted systems.

\section{Home use biosensors}

\subsection{State of the art}

The parameter that continues to set the pace for personal use of biosensors is glucose and it will be used in this chapter to illustrate what can be achieved in minimally-invasive biosensing. The extent of the diabetic market is such that there are considerable commercial and healthcare incentives to drive new developments in monitoring in this field. The WHO statistics from 2004 indicated that there were 170 million diabetics worldwide at that point and lifestyle changes are raising the rate of the development of the condition significantly, with an expected world population of 300 million by 2025 (WHO 2004). The development of portable glucose sensors for diabetics has been reviewed in detail by Newman \& Turner, in 2005, tracing the path of glucose sensing from the Yellow Springs Glucose Analyser developed by Leyland Clark through the introduction of amperometric, mediated glucose sensors that provide reliable and portable glucose sensing up to the 'minimally invasive' sensors on offer today. The frequent blood sampling required by diabetics who use blood testing devices has led to problems for users, including pain and damage to sampling sites, and companies have tried to overcome this by devising better lance systems and looking for alternative sampling sites to the fingertips. Ideally no blood sampling would take place for diabetic home testing and the field is moving towards this.

\subsection{Subcutaneous glucose sensors}

Currently the state of the art in minimally invasive technology is provided by subcutaneous sensors that the user must inject under their skin and clip to a skin mounted transmitter. Systems are available from Medtronic (Guardian ${ }^{\circledR}$ REAL-Time), Abbot (FreeStyle Navigator ${ }^{\circledR}$ ) and Dexcom (Cox, 2009). The sensors can be left in body for up to seven days before removal or replacement and will transmit glucose readings to a meter from the skin mounted transmitter. This is a clear advance in glucose monitoring and the best yet that biosensing has been able to offer the diabetic field.

Other point of care systems are available for some parameters but are all based on blood sampling, such as devices for monitoring of anti-coagulation therapy e.g. HemoSense INRatio meter (Meurin et al., 2009) and lactate measurement devices for sports monitoring and general medicine e.g. Roche Accutrend (Acierno et al., 2008). Thus it is clear that there is no widespread availability of biosensors that are capable of either full or subcutaneous long term implantation and a brief consideration of the reasons for this is appropriate.

\subsection{Device implantation responses}

The host response in the human body to any foreign material is a stimulation of the inflammatory response. Body fluid contact with the device and protein adhesion stimulates cellular activity on the implant surfaces, commencing with leukocyte contact and a cell cascade reaction. This further stimulates protein deposition and fibrous encapsulation of the foreign material, creating a barrier to analyte diffusion and a degradation of device performance. Miniaturisation of devices has not removed these fundamental biological problems. The smaller sensors developed through nanotechnology are not immune to this 
response despite improvements in biomaterials and the use of biocompatible coatings, such as polyethylene glycol, and the use of tissue response modifiers (mainly anti-inflammatory drugs) embedded in devices for local release. Vaddiraju et al., (2010) have reviewed the challenges facing nanosensors for implantation and conclude that while the reduction in size of implant through nanotechnology has lowered the immune response it has not been removed. Nevertheless, they recommend continued research in this field and the development of multianalyte devices for early disease detection.

In addition there may be opportunities to introduce temporary implanted sensors where tissue needs local monitoring over shorter times. An example of such a device is an implantable sensor for cancer marker proteins that can be left in situ during tumour surgery to monitor local tissue response (Daniel et al., 2009). The sensor contains an implanted magnetic label sensitive to cancer markers which can diffuse into the device. It has been demonstrated in a murine model for the monitoring of cancer markers following tumour resectioning. With adjustment of the magnetic label it could equally be deployed for monitoring of metabolites or chemotherapy agents.

Subcutaneous sensors do not fare much better when the host response is considered and are also subject to protein attachment. Gifford et al., (2006) have studied the encapsulation of a subcutaneous needle-type biosensor for glucose using a rat model and concluded that the absorption and infiltration of larger molecules, such as IgG (169 kDa) and serum albumin (66KDa), creates barriers to the diffusion of glucose and is the main cause of loss of sensitivity in these devices. Regular calibration is needed to account for this loss in sensitivity.

\subsection{Less invasive approaches to health monitoring}

If in vivo and subcutaneous biosensors are eventually thwarted by the host response then less invasive methods of obtaining biological samples directly from the subject must be the answer to many diagnostic requirements. There is a great deal of research presently underway to address this. The use of less invasive sensing methods for glucose are explored below, as an example of how minimally invasive monitoring is developing. Methods of noninvasive and continuous glucose monitoring are regularly reviewed (see for example Ferrante do Amaral \& Wolf, 2008; Girardin et al., 2009; Pickup et al., 2005; Tura et al., 2007; Wickramasinghe et al., 2004)

\subsection{Measurement of glucose in body fluids other than blood or interstitial fluid}

Although blood glucose concentrations are of interest, noninvasive methods for measuring glucose have been attempted using a number of different fluids in the body. The following discussion concentrates on fluids that are most readily accessible, such as sweat, saliva, tear fluid and urine while sampling of interstitial fluid will be discussed in later sections. Sweat is an example of a body fluid that is readily accessible through non-invasive means. Glucose levels in sweat have been reported to be similar to glucose levels found in blood. Sweat may therefore represent one option for non invasive measurement (Pellett et al., 1999) of glucose and other parameters. Patches have been developed for sweat collection, and these devices have been trialled for use in the detection of substance abuse (Liberty et al., 2003; Uemura et al., 2004).

The measurement of glucose in urine, urinalysis, has also been used as an indication of blood glucose levels. This has been used clinically for some time and is often the method by which diabetics are first identified (Pickup \& Williams, 1997). Although this method of 
analysis is useful it does not lend itself to continuous, quantitative, blood glucose measurements. Aside from the practical issues of regularly measuring urine glucose levels, storage times in the bladder lead to significant lag times between glucose levels excreted in the urine and those found in the blood. Similarly, glucose levels in other biological fluids, such as saliva, also lag behind blood (Pellett et al., 1999).

Even if this lag time between blood and body fluid glucose was considered insignificant, there are few devices reported in the literature for non-invasive, near continuous, monitoring of glucose levels from sampled human body fluids other than interstitial fluid. An example of a system for human body fluid measurement is a contact lens that reacts to tear glucose levels (Badugu et al., 2003). Contact lenses embedded with a form of boronic acid that contains fluorophores have been investigated as a medium for sensing the amount of glucose in tear fluid. It has been suggested that this approach may be suitable for the continuous monitoring of tear glucose levels, which typically correlate to blood glucose levels. A potential tear glucose operating range of $50 \mu \mathrm{M}-1000 \mu \mathrm{M}$ was reported (Badugu et al., 2003). It has been proposed that users could assess their glucose concentration by comparing the colour of their contact lens against pre-calibrated colour strip (Badugu et al., 2005). Further work is needed to address issues of resolution, lifetime and biocompatibility (Moschou et al., 2004). The main issues concerning this method are, firstly, it seems that glucose fluctuation would only be detected if its concentration increased over what was expected. If this were the case, then the onset of hypoglycaemia would not be detected. The second issue is that this method does not provide a quantitative measure of blood glucose levels so could not be used in conjunction with hypo- or hyper-glycaemic alarms or give indication of insulin dose countermeasures.

\section{Human skin and minimally invasive monitoring}

Due to the potential for access through skin, the majority of approaches taken to minimally invasive blood glucose monitoring have concentrated on this organ. Skin is an effective barrier to the transport of molecules into the body or out of the body, due to the structure of the dermis, epidermis and stratum corneum, but does allow some molecular transport, interstial fluid collection and subcutaneous access. The remainder of the chapter will deal with methods of non-invasive monitoring based on dermal or transdermal analysis of the analytes that can be obtained through the skin.

\subsection{Dermal monitoring approaches}

\subsubsection{Non-invasive - electromagnetic analysis}

Electromagnetic radiation provides the possibility for truly non-invasive glucose measurement with a very low risk of adverse side effects. Electromagnetic (EM) wave radiation can be observed over a wide range of different wavelengths. The range of wavelengths gives rise to the electromagnetic spectrum as shown in Figure 3.

Electromagnetic radiation will interact with molecules and atoms. These energetic interactions can be used to probe glucose, and potentially other parameters, in various ways depending on the chosen wavelength. As sensing of blood glucose has to be non-harmful to the body, shorter wavelengths than the optical region cannot be used as radiation below these wavelengths becomes ionising.

Optical methods (between the visible and far-infrared parts of the electromagnetic spectrum) are largely based upon focusing a beam of light onto a tissue test site and measuring how the 
light is modified by the target tissue. Light will interact with biological tissues in a number ways including absorption, reflection, scattering, transmission, polarisation or modification in wavelength. The nature of this interaction and the degree to which it occurs will depend upon the components of the tissue (e.g. water, fat, glucose) and their respective concentrations within the target sample. These compounds have recognisable optical signature responses to incident light and many forms of analysis have been investigated to relate these optical signatures to the concentration of glucose in tissue samples.

When the glucose concentration of the sample site has been measured, this then has to be related to blood glucose concentration. This is not a straightforward process as within a chosen sample site there will be a number of compartments each containing a different concentration of glucose. Each of these different compartments will contribute to the measured signal. For sites chosen on the skin, the signal is likely to be dominated by the compartments containing intracellular fluid, interstitial fluid and capillary blood. The ratio of these three groups will also vary depending on site location leading to very site specific measurement calibrations.

Two of the most popular areas for investigation of glucose by electromagnetic spectroscopy are near-infrared spectroscopy and mid-infrared spectroscopy.

\subsubsection{Near-Infrared Spectroscopy (NIR)}

The near-infrared range used for investigating blood glucose spans an electromagnetic wavelength range of approximately $1000 \mathrm{~nm}-2500 \mathrm{~nm}$. This region is a very popular range for investigation of glucose as it permits penetration into deep tissue $(\sim 1-100 \mathrm{~mm})$ depending on the chosen wavelength. The depth of penetration will decrease with increasing

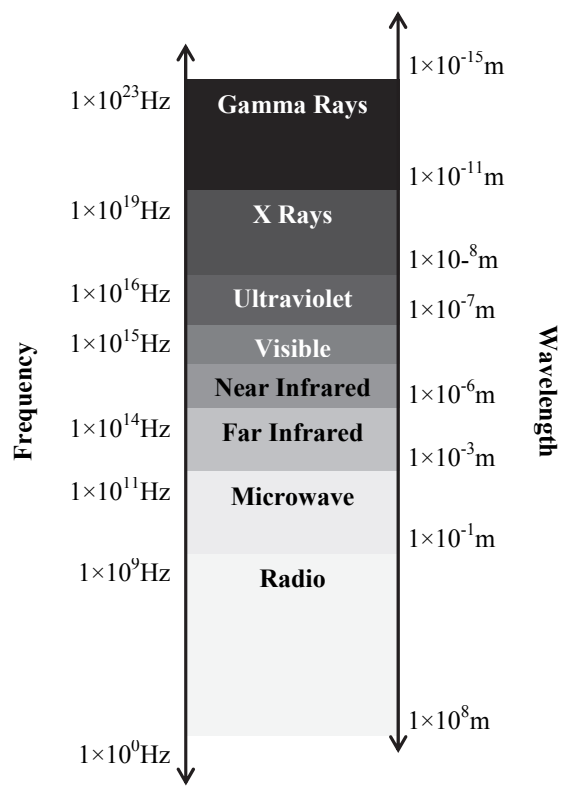

Fig. 3. An illustration of the regions of the electromagnetic spectrum with approximate corresponding frequency and wavelength. 
wavelength. Another advantage is that the absorption coefficient of water is weaker in nearinfrared region when compared with the mid-infrared region

There are challenges to glucose measurement by near-infrared spectroscopy and a variety of tissue test sites have been researched (Oliver et al., 2009). From a physiological perspective, the challenges are compound. Firstly this arises from the relatively low concentrations of glucose in the body when compared with the presence of other substances that affect the NIR signal such as fats and water. Secondly, the absorption coefficient of glucose is low in the near-infrared region and, finally, the spectral bands due to glucose overlap with bands due to water, fat, haemoglobin and proteins. This introduces significant technical challenges for signal sensitivity and signal interference, which will need to be addressed in order to demonstrate sufficient accuracy of glucose measurement (Pickup et al., 2005; Tura et al., 2007; Ferrante do Amaral \& Wolf, 2008). The cost and physical size of equipment required for infrared spectroscopy measurements may also limit its application in continuous monitoring, particularly in the context of wearable sensors. Despite these challenges, there is still a large amount of commercial activity in this area.

\subsubsection{Mid-infrared region}

The mid-infrared region can span between $2500 \mathrm{~nm}-10,000 \mathrm{~nm}$. As this radiation is at longer wavelengths than Near-Infra Red, the depth of penetration is reduced to the micrometer range (Tura et al., 2007). At these wavelengths, less of the light is scattered and the majority of light is absorbed. In this region the absorption peaks are sharper and more defined when compared with the broader weaker peaks seen in the NIR region. This is observed for both glucose and other compounds (Ferrante do Amaral \& Wolf, 2008). However, hydration level of the skin can has a strong impact on such absorption signals and this is subject to variation.

\subsection{Transdermal monitoring approaches}

The transdermal route has clear attractions and can be divided into two approaches: interstitial fluid sampling, and transdermal extraction. In the first method, the intention is to directly sample interstitial fluid and this can be done via microneedle technology, sonophoresis or thermal ablation. For the second approach, transdermal extraction of molecules is achieved by electrically sampling the skin interface. This largely makes use of the existing skin transport routes in the hair follicles, sweat glands and in nano and micoporous structures in the skin. This is the realm of iontophoresis and electroporation. Both approaches could lend themselves to combined extraction and sensing using micro and nano electromechanical systems (MEMS and NEMS technology) and therefore should be of interest to those developing miniaturised sensors. Much of the literature in the field has been generated from research into transdermal drug delivery, but it is equally applicable to the extraction of molecules via the skin. Arora et al., (2008) comprehensively review Microsystems for transdermal drug delivery and describe state of the art skin delivery techniques that could easily be adapted for the collection of, or access to, interstitial fluid. They point out that creating micrometre-scale breaches in the skin is well tolerated and the skin will recover quickly from such breaches. Thus, it is possible to temporarily reduce the skin barrier for molecular collection and analysis. This means that the accepted passive limits of drug delivery (or molecular extraction) of $500 \mathrm{Da}$ can be surpassed and large molecules could be detected as well as small. 


\subsubsection{Interstitial Fluid Sampling (ISF)}

The most easily understood of the device types for transdermal extraction are microneedle devices. Microneedle arrays can be fabricated and pressed onto skin or scraped over it to create the necessary breach in the stratum corneum. Successful application of a microneedle array can increase skin permeability in that region by up to 4 orders of magnitude. Microneedles can be solid or hollow and therefore such devices could offer a degree of flexibility in terms of location of biosensors. Combined sensors and microneedles are already being suggested by some groups. For example, Mukerjee et al., (2004) demonstrated successful collection of interstitial fluid from a microneedle and capillary device which enabled glucose measurement. Further investigation of how interstitial fluid levels of specific analytes compare with blood levels is required, but early signs are encouraging. Mitragotri et al., (2000) also investigated a range of other parameters in rats, with ISF collected by sonophoresis taken together with simultaneously collected serum samples, and found good agreement between glucose, albumin and triglycerides in ISF and serum, but higher than expected lactate and calcium in the ISF as compared to serum.

\subsubsection{Ultrasound (sonophresis/phonophoresis)}

Ultrasound has been explored as a method for enhancing drug transport across the skin. Various power levels, duty cycles, and frequencies have been examined. Drug delivery for a range of hydrophilic and hydrophobic compounds enhanced by sonophoresis has been comprehensively reviewed by Escobar-Chavez et al., who conclude that the use of sonophoresis in skin permeation enhancement and drug delivery is likely to increase (Escobar-Chavez et al., 2009)

Various frequencies of ultrasound can be chosen, from low frequencies $(20 \mathrm{kHz})$ to very high frequencies (low $\mathrm{MHz}$ ), to be applied to the skin to enhance permeability. It has been suggested that in the lower frequency range (approximately $20-90 \mathrm{kHz}$ ) there exists a threshold intensity below which no detectable skin permeability enhancement will be observed. This threshold intensity increased with frequency (Tezel et al., 2001). It has also been suggested by the same authors that low frequencies $(20 \mathrm{kHz})$ induced localised transport compared to a more dispersed effect seen with higher frequencies $(58.9 \mathrm{kHz})$. Other authors (Ueda et al., 1995) have suggested that high frequency (10 and $16 \mathrm{MHz}$ ) ultrasonication can concentrate the ultrasonic energy on the stratum corneum in vivo. They also reported that electron microscopy indicated that the intercellular route of the stratum corneum is influenced by ultrasonication at these frequencies.

Cavitation, the growth and collapse of gas bubbles, is generally reported to be the dominant mechanism of sonophoresis. Cavitation is thought to disorder the lipid bilayers in the stratum corneum creating mass transfer pathways and thus increasing the diffusion coefficient of solutes. However, it has been suggested that cavitation alone cannot account for the total enhancement effect observed (Cancel et al., 2004). Several mechanisms have been suggested to contribute to this transport phenomenon. Among these are structural changes caused by cavitation, thermal effects, mixing in the liquid phase and acoustic streaming through hair follicles and sweat ducts. In addition, a convective mechanism of enhancement has also been suggested, although no quantitative analysis has been proposed. Cavitational and mechanical effects increase tissue temperature and skin permeability is increased by an increase in temperature. The ultrasonic apparatus used in one study (Ueda et al., 1995), raised the temperature in the donor compartment (in vitro) by $3-4^{\circ} \mathrm{C}$ during the application of ultrasound. This may account for some reversible effects accompanying 
ultrasound. In vivo, raising tissue temperature should not exceed an increase of $1^{\circ} \mathrm{C}$ as anything above this can cause irreversible damage to tissue. Such unwanted effects may account for the irreversible effects observed such as a decrease in barrier function of stratum corneum.

Successful application of ultrasound to skin for the sampling of interstitial fluid and detection of glucose has already been successfully demonstrated by Kost et al., (2000) and it remains a topic of research interest.

In summary, the sonophoresis approach to skin permeability enhancement is clearly of interest although physical size and cost of ultrasound based enhancement technology may limit its use in the field of continuous monitoring, particularly with application to wearable sensors.

\subsubsection{Radiofrequency (RF) thermal ablation}

Radiofrequency thermal ablation has been used in microsurgery for treatment of conditions such as tumours. This method consists of an array of needle like electrodes that are placed on the skin which deliver heat that kills the tumour while leaving the surrounding healthy tissue unharmed. This method has been tested in a similar way for aiding transdermal drug delivery. This works by using high voltage radiofrequency currents to create aqueous microchannels in the skin (Sintov et al., 2003). This study examined this effect in vitro (across porcine skin) and in vivo in rats. The study reports increased delivery of the chosen drug (diclofenac) by a factor of 30 compared with the control over a 12 hour period. As RF thermal ablation has been demonstrated an effective method of increasing skin permeability, it is conceivable that it could be adapted for use in transdermal extraction or assisted diffusion.

\subsubsection{Electroporation}

Electroporation refers to the application of high electric fields for short bursts to skin causing the formation of micropores in the skin. A cell bilayer can be electroporated by the application of transmembrane voltages in the range $0.3-1 \mathrm{~V}$ and thus $1 \mathrm{~ms}$ pulses of between $100-$ and $1500 \mathrm{~V}$ have been used to electroporate the stratum corneum which contains approximately 100 bilayers (Vanbever \& Preat, 1999). However, there is evidence that even application of moderate voltages up to $60 \mathrm{~V}$ across the skin causes electroporation (Chizmadzhev et al., 1998) and thus it should be expected that some poration will occur during iontophoresis. The formation of micron feature openings in the SC by electroporation leads to the possibility of extraction of higher molecular weight molecules. There is considerable scope for localised electroporation of skin by microdevices and microelectrodes that is yet to be exploited.

\subsection{5 lontophoresis}

Iontophoresis is a process where two electrodes, with good interface conductivity (such as silver chloride and conducting gel), are placed upon a membrane and a small voltage is applied to deliver a low current through the membrane (or skin in human applications). This voltage usually seeks to drive a constant DC current in the range of $300-500 \mu \mathrm{A} / \mathrm{cm}^{2}$. This method can be used to enhance transport of ionic elements, or molecules and compounds through the skin as a result of the interaction of the charged ions and molecules with the imposed electric field. Uncharged molecules are also transported by 
electroosmosis. The skin is a permselective membrane that, at physiological $\mathrm{pH}(\sim 5.0-6.0)$, supports a net negative charge (Manabe et al., 2000). As a result, a positively charged ion will penetrate more easily across the skin than a comparably sized negative ion. An electroosmotic solvent flow will also be established. This electroosmotic effect predominates in the anode to cathode direction because current is preferentially carried by cations and momentum is transferred to the solvent by cation movement. This momentum causes a net convective flow (electroosmosis) from anode to cathode and, as a result, there will be enhanced transport of dissolved, uncharged solutes in the same direction.

Iontophoresis may also enhance the transdermal movement of larger molecules (> $1000 \mathrm{Da})$ (Delgado-Charro et al., 1994). However, the transport numbers of such molecules (i.e., the fraction of the total current flowing which is carried by the large molecule of interest) are very small because of competition from smaller, more mobile ions such as the background electrolytes and/or receptor solutions. Research in this field is ongoing and unexpected behaviour has sometimes been reported with larger molecules such as peptides. For example, a very weak dependence of flux upon applied current density, and even an inverse relationship between transport and applied peptide concentration, has been observed (Delgado-Charro et al., 1994).

\section{Minimally invasive monitoring by reverse iontophoresis}

Reverse iontophoresis is where the application of electric current across the skin is used to extract a substance of interest from within or beneath the skin to the surface (Santy \& Guy, 1996a,b). Figure 4 illustrates the application of a constant current via two skin mounted electrodes. The electrodes are housed in electrically conducting gel chambers. The diagram also illustrates the resultant solvent flow that is generated. Circles with a ' + ' represent positive ions and circles with a '-' represent negative ions. Circles with a ' $G$ ' represent the glucose that is caught in the solvent flow and carried into the gel chamber for analysis via an imbedded sensor.

Transdermal molecular extraction by reverse iontophoresis has a distinct appeal as it is an electronically controlled and programmable method of extraction, that can be turned on and off at different points in the diagnostic cycle. Because there is no deliberate breach of the skin there are four separate routes of molecular transmission that are available for molecular transport; transcellular, intercellular, via hair follicles and via eccrine (sweat) glands (Riviere \& Heit, 1997). The mechanism of extraction is non-specific; there are a great number of potential analytes that could be measured and therefore a great number of potential uses for reverse iontophoresis. An example of this has been shown by Sieg et al., where glucose and urea were simultaneously extracted (Sieg et al., 2004b). We have demonstrated good levels of simultaneous lactate and glucose extraction in healthy volunteers by application of iontophoresic current of $300 \mathrm{uA} \mathrm{cm} \mathrm{cm}^{-2}$ in 15 minute cycles for periods up to 1 hour as shown in Figure 5 (Ching \& Connolly, 2008b). Others have shown the simultaneous extraction of a range of amino acids in human subjects (Sieg et al., 2009).

Investigations into methods of optimising the analyte extraction have revealed that cathodic extraction is enhanced as $\mathrm{pH}$ increases as far as can be feasibly maintained in contact with the skin surface; the reverse is true for anodal extraction (Santi \& Guy, 1996b). In-vitro, electroosmosis increases with decreasing ionic strength in the electrode chambers. However, sufficient electrolyte must be present to sustain the necessary electrochemical reactions occurring at the electrode surfaces. 


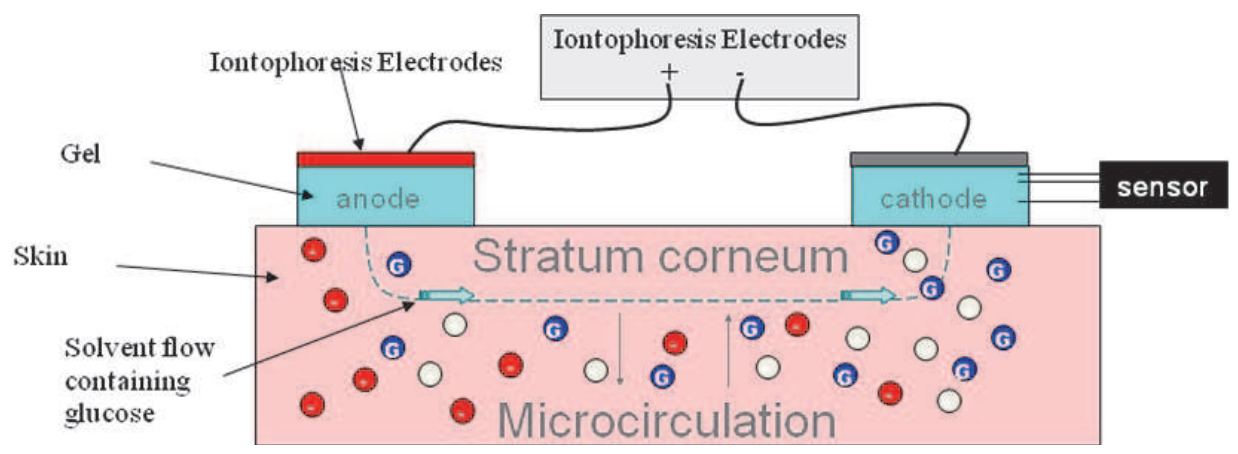

Fig. 4. Schematic illustration of the principle of reverse iontophoresis showing an iontophoresis extraction device supplying a constant current to an anode and cathode.
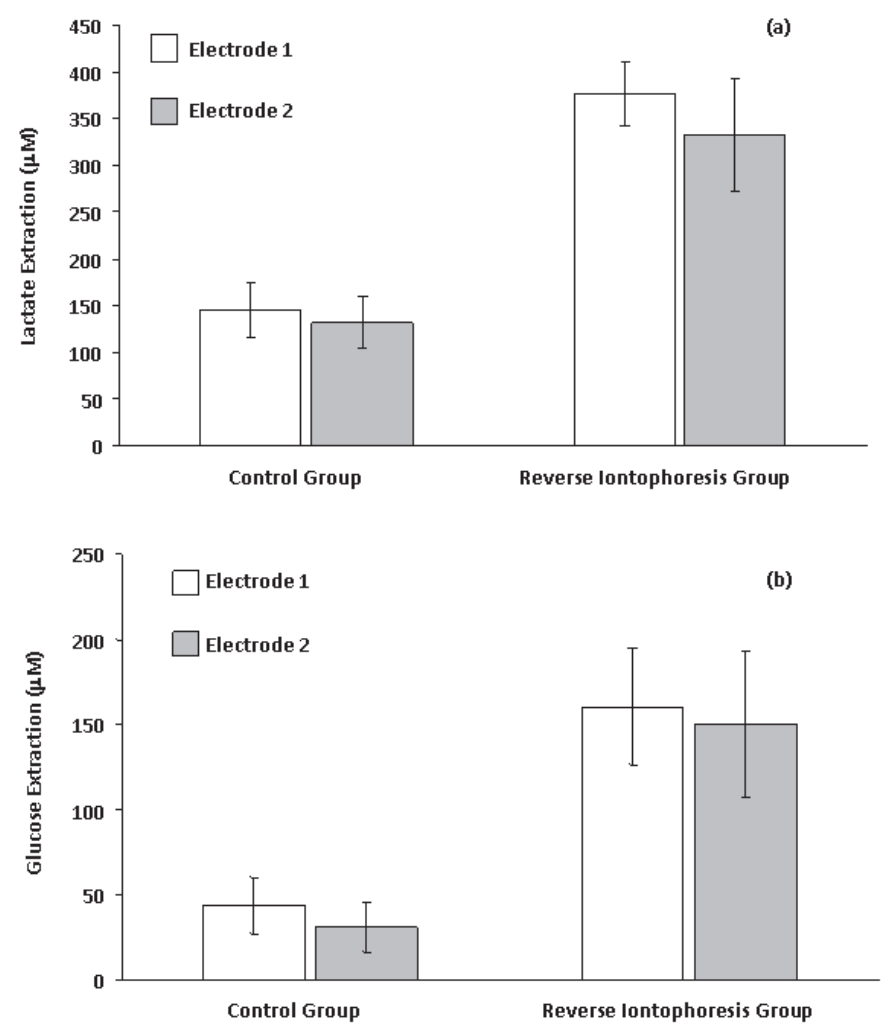

Fig. 5. Average results of long duration bipolar direct current application (current density of $0.3 \mathrm{~mA} / \mathrm{cm}^{2}$, polarity of electrodes reversed at intervals of 15 minutes, experimental time of 60 minutes) on human transdermal extraction of (a) lactate and (b) glucose (mean \pm SD; $\mathrm{n}=10$ ). Extraction of lactate or glucose by reverse iontophoresis was significantly higher ( $p<0.001$ for both cases) than that of the control sample. Reprinted from Ching \& Connolly, $2008 \mathrm{~b}$ with permission. 
Further work by Santi \& Guy used a non-metabolizable sugar mannitol as a marker and found that decreasing the $\mathrm{pH}$ in the anodal chamber and increasing that in the cathode chamber improved the total quantity of electroosmotic flow from beneath the skin (Santi \& Guy, 1996a). The authors also fixed $\mathrm{pH}$, and reduced the ionic strength of the electrode chamber solutions (relative to the physiological level), confirming again enhanced reverse extraction, most notably at the cathode.

\subsection{Effects of iontophoresis on human skin and skin recovery}

The successful clinical application of iontophoresis will require minimal or no side effects as well as the rapid recovery of the skin barrier after the current flow has been terminated. Curdy et al., (2001) has reviewed non-invasive methods for skin integrity assessment. These methods include Transepidermal water loss (TEWL), Impedance spectroscopy (IS), Attenuated total reflectance-Fourier transform infrared (ATR-FTIR) spectroscopy and Laser Doppler flowmetry (or velocimetry) (LDF). Curdy et al., used a variety of these methods to assess skin function following iontophoresis in vivo (Curdy et al., 2002). The paper demonstrated a reduction in skin barrier impedance, as desired and expected, postiontophoresis. However, the paper concludes that there is no evidence of an association between the observed reduction in impedance and skin damage.

The potential for reverse iontophoresis as a technology for transdermal extraction has been most noticeably demonstrated by the Glucowatch (Cygnus Inc). Significantly, the GlucoWatch gained FDA approval for diabetic monitoring in 2001. The FDA approval was based on nine pivotal clinical studies, seven assessed the effectiveness of the device and two assessed safety. A summary of these studies and the criteria on which the GlucoWatch achieved FDA approval can be found in the relevant FDA approval documentation (Summary of safety and effectiveness data, PMA No. P990026, FDA 2011). In 2002, the second generation device (GlucoWatch G2) obtained FDA approval (Summary of safety and effectiveness data, PMA No. P990026S008b, FDA 2011) Further details on this device can be found in the literature (see for example Tierney et al., 2001). Despite obtaining FDA approval in 2001 the GlucoWatch did not secure market adoption. Technical or user-related issues, such as sweating on the skin under the device causing a short circuit that (when detected by two electrodes specifically designed for this) stopped glucose readings, impeded its widespread uptake

\subsection{Reverse iontophoresis challenges}

If reverse iontophoresis is going to make the impact that many expect in the field of minimally invasive monitoring, it is clear that key technical challenges will have to be overcome. It is now worth exploring each in some detail and examining what research is underway to address each challenge. The following analysis is largely based on glucose sensing, although other parameters are mentioned where appropriate. However, many of the technology challenges are common across a range of potential parameters, and therefore the discussion should be of relevance to all readers engaged in minimally-invasive sensor development.

\subsubsection{Comfort}

It is clear that one of the major advantages of minimally invasive sensors is an ability to perform monitoring that is relatively pain free. This is clearly understood when the 
replacement of frequent, fingerstick blood sampling is considered. Any device that can provide the same information without the requirement for blood samples would represent a real advance and facilitate better disease management strategies, which are known to reduce secondary complications of diabetes (UK Prospective Diabetes Study Group (1998a; 1998b; 1998c; 1998d)). It is therefore essential that any proposed technology does not in itself introduce new barriers to adoption, such as pain or inconvenience associated with its use. The level of pain experienced during iontophoresis is related to the current density, and it is generally accepted that currents in excess of $300 \mu \mathrm{A} / \mathrm{cm}^{2}$ cannot be readily tolerated for extended periods (Ledger, 1992). This places limits on the type of molecule that can be extracted transdermally for analysis, with molecules over 500Da being unlikely to cross the skin at this level of current. In addition, the applied current density has also been directly correlated with the transdermal flux (Delgado-Charro et al., 1994). Using the neutral molecule, Mannitol, Delgado-Charro et al., were able to demonstrate that flux was linearly correlated with applied current density over a range of $0.14-0.55 \mathrm{~mA} / \mathrm{cm}^{2}$ (DelgadoCharro et al., 1994). Therefore, any efforts to reduce the current level must be balanced with the need to extract a quantifiable amount of the analyte of interest, and for biosensing this is limited by the range of the sensor to be deployed in the gel electrode. Consequently, technologies that can enable efficient transdermal extraction at low current levels are particularly appealing

Since one of the potential benefits of minimally invasive sensors is the ability to monitor continuously over extended periods, it is worth examining in more detail how the skin interface responds to the process of reverse intophoresis over prolonged extraction periods. On a practical level, there is evidence of localised skin irritation over prolonged durations of reverse iontophoresis (Howard et al., 1995). There are two main reasons for this beyond the localized heating that can occur at higher levels of current. Firstly, the use of direct current (DC) and secondly, the use of embedded biosensors within the skin gel.

Direct current (DC) is believed to generate high concentrations of hydroxyl ions within the anodal gel compartment, with the production of hydrogen ions within the cathodal compartment. Since both gel compartments are in intimate contact with the skin surface, the resultant localized alterations in $\mathrm{pH}$ may be, at least in part, responsible for the erythema and stinging that has been reported in some studies (Howard et al., 1995). In efforts to address this, polarity reversal has been introduced. Here, the polarity of the electrodes is regularly alternated, such that the current flow changes direction. In addition to reducing the effects of local $\mathrm{pH}$ imbalances, it has also been shown in several studies, including our own, to actually enhance iontophoretic transport (Ching et al., 2008a). DC current can cause electrical polarization of the skin, thus inhibiting molecular transport across it, and it has been proposed that the enhanced transport, produced by switching electrode polarity, is likely due to a reversal of this skin polarisation process (Ching et al., 2008a). It is worth mentioning, however, that not all studies have demonstrated such an enhancement effect on transdermal flux (Santi \& Guy, 1996a; Santi \& Guy, 1996b). It is likely that the optimum extraction conditions are molecule dependent. Careful consideration of current level, duration, and delivery mode are therefore required.

Another factor to be considered in transdermal extraction is the accumulation of reaction intermediates and products resulting from the detection technology. The extent to which this represents a barrier to clinical adoption depends on the molecule of interest, and how it is to be measured. If one considers the detection of electrolytes collected at the skin surface by reverse iontophoresis, a relatively simple embedded ion-selective potentiometric sensor 
could be used to measure the ion concentration without the production of any deleterious reaction products. However, the situation was quite different with the first generation of reverse-iontophoresis based continuous glucose monitors. The biosensors employed in these devices used glucose oxidase enzyme that was distributed throughout the gel (Tierney et al., 2001). Considering that the product of the glucose-glucose oxidase reaction is hydrogen peroxide, a known irritant (Watt et al., 2004), it is clear that irritation is a risk with prolonged use of these types of embedded sensors. The use of mediated enzyme electrodes for analyte detection, where the detection enzyme is bonded to the electrode alongside a mediator (Liu \& Okuma, 1998; Sato et al., 2006), or where the components are dispersed in the ink of a printed electrode (Saby et al., 1995), has removed this particular limitation. These mediated biosensors, which do not produce hydrogen peroxide, are now the most common method of glucose detection used today.

At this stage, it is worth repeating that one of the main aims of minimally invasive sensors, is to replace existing invasive measurement methods where this will be of real clinical benefit. If this replacement is to be justified, then the proposed system must provide the clinician or patient with at least the same level of information as the original test format. The utility of reverse iontophoresis therefore relies, at least in part, on the ability to use the reading obtained at the skin surface as an indicator of the concentration of the analyte of interest in the blood. This is a key challenge, and many view it as the Achilles heel of the technology. It is therefore worthy of discussion and we will examine some of the technical challenges that have hampered efforts to establish robust correlations between blood and skin levels of an analyte.

\subsubsection{Lag}

A significant criticism of transdermal technologies that rely on sampling interstitial fluid, such as reverse iontophoresis, is that there is a lag time in detection of the molecule at the skin interface (Kulcu et al., 2003). It is known that blood glucose changes can occur rapidly in the blood (Pickup \& Williams, 1997). A lag time of around 20 minutes was reported for the GlucoWatch (Tamada et al., 1995) although other studies suggest that shorter lag times of around $5 \mathrm{~min}$ may be possible (Kurnik et al., 1998). So it is not yet clear that lag time is an insurmountable problem for reverse iontophoresis approaches. It is also worth noting, that the relative importance of lag time can be viewed as being dependent on the molecule of interest. Given that attention has largely focused on glucose measurement thus far, it is perhaps not surprising that this problem has received considerable attention. However, there are other applications where the impact of lag time would present a much less significant problem. When one considers therapeutic drug monitoring for instance, a reverse iontophoresis patch may be applied to the skin for several hours before a measurement is made to estimate the final concentration of the drug within the blood (Leboulanger et al., 2004). Similarly, it is of little apparent clinical benefit to measure disease marker molecules continuously, or over an extended duration. Rather, the purpose of such detection would be to provide a snapshot to inform diagnosis, enabling treatment or prevention measures to be initiated. It is therefore clear that one must consider the molecule, and the intended use of the information, since both will impact the extent to which existing reverse iontohporesis technologies can usefully be applied. The point has already been made earlier in this chapter, but this reinforces the value of clinician input at the very earliest stages of device development. 


\subsubsection{Gel properties}

Up until this point, our discussion on lag has focused on what is happening within the different compartments of the body, notably the relationship between blood and interstitial fluid analyte concentrations. The following discussion now considers what happens within the gel that collects the molecule at the skin surface. There are several challenges associated with this aspect of the technology. The first relates to irritation and this has been covered in previously in the text. The second relates to the diffusion profile within the gel environment and ultimately the systems sensitivity. The diffusion characteristics of small molecules within solutions and gels are relatively well described in the literature (Wesselingh, 1993; Westrin et al., 1994). However, the impact of molecular diffusion within the gels used in reverse iontophoresis has so far been examined in only a limited number of studies (Künzelmann \& Böttcher, 1997; Kurnik et al., 1998).

Let us first consider what happens within existing reverse iontophoresis gels. The molecule of interest is extracted across the skin and collects in a gel reservoir. At some point following application of the current, the molecule will have reached a level that is detectable using an embedded sensor. The first point to make is that this introduces a lag time, meaning that the sensor response will be delayed. Figure 6 illustrates this concept in practice for a hydroxypropylmethylcellulose gel reservoir of approximately $5 \mathrm{~mm}$ depth, with an embedded glucose biosensor at the bottom of the gel reservoir which we developed for testing gel behaviour (McColl, 2001). The time between addition of glucose to the gel surface and the first sensor response can be as much as one hour (Fig 6(b)). The use of a reduced gel concentration, with a consequent increase in the diffusion coefficient (from 2.44 $\times 10^{-11}$ to $6.87 \times 10^{-11} \mathrm{~m}^{2} / \mathrm{s}$ ), reduces this lag time to around 30 minutes (Fig6 (a)).

The time required for accurate detection will be related to gel volume, diffusion coefficient, molecule size and mobility, and gel thickness. Since the properties of the parameter of interest are necessarily fixed, modification of the gel properties is the main option for improving detection performance. We have shown in Figure 6 that diffusion time can be reduced by selecting a gel with a high diffusion coefficient. In addition, reducing gel volume and thickness will also reduce lag time. Indeed, lag times of 5-10 minutes were reported in an experimental study, which examined glucose diffusion in the type of thin gel layers $(127 \mu \mathrm{m})$ used in the Glucowatch (Kurnik et al., 1998). Future designs are likely to make use of gel printing technology which will enable the manufacture of very thin gel layers to reduce measurement lag time. This will also lead to enhanced sensitivity by reducing the dilution of the molecule in the gel reservoir. Taken together with the developments in enhanced skin permeation methods described earlier, advances in this area may help pave the way for near zero current, diffusion dominated transdermal extraction systems.

A second important element of diffusion in transdermal systems is the diffusion profile of the analyte to the sensor. It is important to work on the sensor design to enhance diffusion sensor profiles at the sensor interface and to calibrate the sensor carefully in situ. The gel improvements described above may be sufficient for the continuous detection of molecules where the concentration is relatively unchanging over short time periods (hours). As an example, it can be envisaged that for a molecule of relatively constant concentration, such as sodium, measurement within the gel could take place after a specified time, $t$, and the blood concentration inferred by a relatively simple algorithm based on an initial calibration. However, for molecules such as glucose, there is high variability in the blood levels of the molecule which can change rapidly over a period of minutes (Pickup and Williams, 1997). Since the diffusion profile of the molecule through the gel is essentially uncontrolled in 


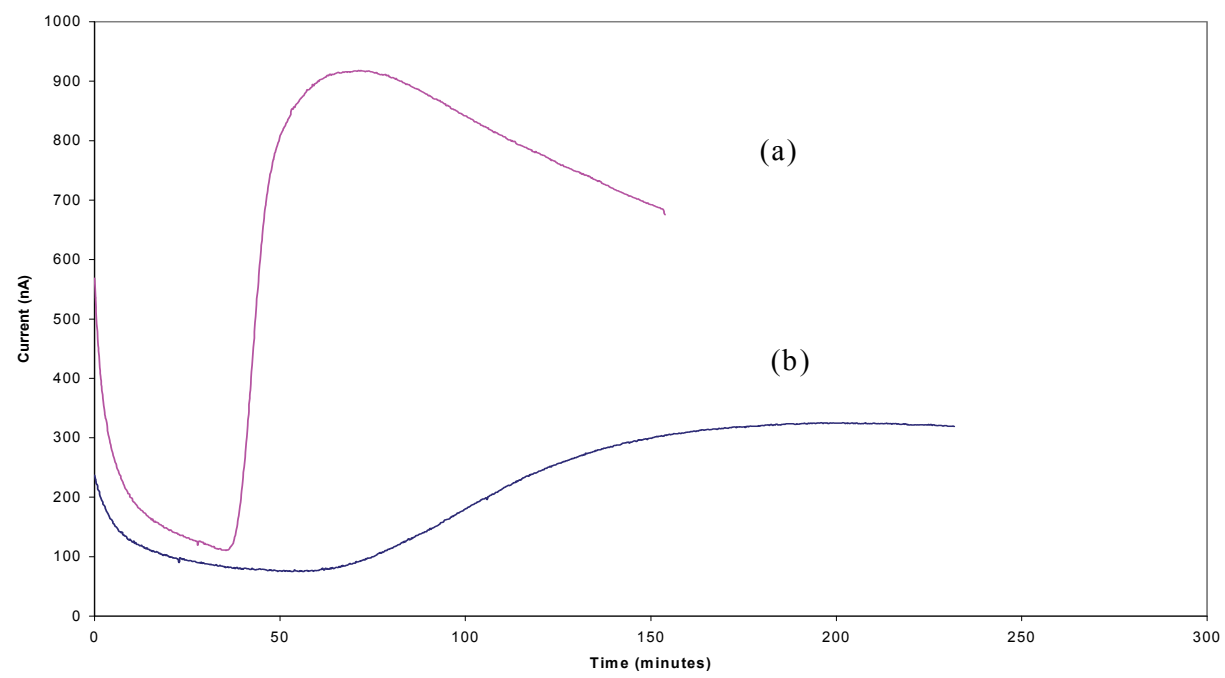

Fig. 6. Anodic current recorded from 2 individual electrodes in 2\% HPMC gel (a) and 4\% HPMC gel (b). At time zero, glucose was added to one end of the gel slab. The profile indicates the glucose dependent amperometric response observed at a sensor positioned at the opposite end of the gel slab.

existing systems, this means that continuous measurement and responses to rapid reductions/increases are difficult to measure. It is in this context that more sophisticated gel extraction systems are called for in the future.

\subsubsection{Calibration}

Whatever the parameter to be measured, it is clear that what is measured at the skin surface must provide an indication of the blood level of the molecule. Most reverse iontophoresis-based detection systems developed to date have relied on calibration of the device with the use of a finger stick blood sample. In recent years, research effort has been dedicated to the use of what is collectively known as an 'internal standard'. The basic requirement for an internal standard is that it must be small enough to be extracted using reverse iontophoresis, and that its concentration within blood should be relatively unchanging, and independent of the molecule of interest. The internal standard was first proposed in 1993 (Numajiri et al., 1993). Since then, there have been several alternative molecules proposed (Leboulanger et al., 2004; Sieg et al., 2004a; Sieg et al., 2003; Sieg et al., $2004 \mathrm{~b}$ ). Of these, the most widely studied to date has been sodium. Sodium is a small, and highly mobile, cation that is present in blood at a concentration of around $133 \mathrm{mM}$. It has been examined as an internal standard for glucose measurement calibration in a previous in vitro study (Sieg et al., 2003). Using excised porcine ear skin, it was shown that the ratio of sodium to glucose fluxes was correlated to the concentration of glucose within the test chamber, indicating the potential utility of sodium to act as an internal standard for sample free glucose calibration. However, in the subsequent follow up study in a selected set of human volunteers $(\mathrm{n}=12)$ it was found that the promising correlations observed in vitro were not replicated in vivo, with the authors concluding that, "sodium, as the major charge carrier across the skin is not very sensitive to relatively subtle differences in skin 
charge. As such it is a less than ideal internal standard for glucose monitoring."(Sieg et al., 2004a). In the same study, it was reported that potassium flux varied among individuals and within the same experiment although no data were provided. The reasons for the apparent failure of sodium as an internal standard remain to be elucidated, and we will return to this later in this chapter.

\subsection{Reverse lontophoresis - future prospects}

In the above discussion, we have outlined limitations around comfort, irritation, sensitivity, and calibration with reverse iontophoresis systems developed to date. We will now go on to describe some of the most recent technical solutions that are being developed in response to these limitations.

There are several possible approaches whereby the current level used in reverse iontophoresis may be reduced. One potential method of achieving this is through the use of permeability enhancers. If the permeability of the skin is increased then a greater flux of the analyte can move across the skin for detection. In the case of reverse iontophoresis, this provides the opportunity for a reduction in the amount of current required for analyte extraction. There are many methods that can be used to achieve this, including chemical, mechanical, and electrical permeation enhancement methods. There are many different chemical enhancers used, with varying mechanisms of action. It is beyond the scope of the present discussion to go into each method in detail, and the reader is referred to a review of the area by Thong et al., 2007, which outlines the range of chemicals commonly used, and provides a list of FDA approved skin patches utilizing this approach for transdermal drug delivery (Thong et al., 2007). Mechanical permeation enhancement has been described previously in the text and includes: ultrasound; radiofrequency ablation; and epidermal tape stripping (Lademann \& Hadzija, 2009; Ruddy et al., 1995). Iontophoresis and Electroporation lie within the realm of electrical permeation methods and have been also been described elsewhere. The synergistic use of a variety of the above enhancement methods has been well documented in the literature (Mitragotri, 2000).

In identifying further opportunities for improvements to transdermal technologies, and minimally invasive sensing more generally, it is instructive to revisit the concept of the internal standard and to consider the reasons why no candidate has yet proven successful in a commercial device. We have previously described the in vivo study by Sieg et al., 2004a, which demonstrated that sodium could not reliably be used as an internal standard for glucose measurement by reverse iontophoresis. Despite sodium flux being constant across all subjects, the glucose flux was an order of magnitude lower in 4 out of the 12 subjects studied. In addition, the constant sodium flux observed in the same subject over the course of a year did not reflect the high variation in glucose flux observed, over the same period in the same subject. Specifically, large decreases in electroosmosis were observed in the winter months. One potential explanation for the discrepancy between glucose and sodium fluxes is that the barrier properties of the skin are not constant between subjects and can indeed be variable from day to day within the same person. The question is therefore, how can such variations in skin permeability to different molecules be controlled for?

\subsubsection{Skin permeability}

Skin is a highly complex barrier, and it is well known that human skin permeability is highly variable (Cevc \& Vierl, 2010; Cornwell \& Barry, 1995). Large differences in 
permeability can be observed between different body sites on an individual. In addition to this intra-individual variability there also exists an additional large inter-individual variability. For example, significant differences exist between individuals of different race, age, gender etc. Differences in skin permeability can be at least partially explained by structural factors such as stratum corneum thickness, intercellular lipid composition and the density of skin appendages. In vivo, biological factors such as skin metabolism and skin surface temperature may also have an impact. Finally, as well as naturally existing skin differences, individuals treat their skin with a variety of soaps moisturisers and other cosmetics. Given that skin permeability is crucial for reverse iontophoresic transport, and that this parameter is highly variable, then it is highly likely that accurate calibration will only be achieved by controlling for this variable.

There are a variety of non- or minimally-invasive methods that are available to characterise variations in skin properties. These have been the subject of review elsewhere, and have been summarized in this current chapter. A detailed description of the routes of in-vivo molecular transport across human skin, with a focus on the influence of iontophoresis was provided by Riveiere \& Heit, 1997. The following discussion will consider the utility of impedance measurements, and will be focused within the context of calibrating transdermal extraction processes. The discussion will then be broadened out to the use of impedance in other minimally invasive monitoring and diagnostic applications, including hydration monitoring, and cancerous tissue detection, before concluding with a brief exploration of other similar technologies for measuring tissue properties minimally invasively which are useful in diagnostics and patient monitoring.

The human body is made up of trillions of cells containing, and surrounded by, electrolyte solutions of multiple cations and anions (sodium and chloride are the most abundant). The lipid membranes of these cells together with the proteins and fats of the body form a complex electrical domain worthy of study and characterization in the quest to understand person to person tissue variability.

\subsubsection{Skin impedance calibration}

The electrical impedance of the skin is dominated by two components, resistance and capacitance. Skin impedance is represented mathematically as a phasor quantity, or as two components represented by real and imaginary numbers. In its simplest form we can think of the real term as accounting for resistive elements in the skin, and the imaginary term as largely representing capacitative features of the tissue. It is a field of study in its own right and the reader is referred to two review articles, which serve as a useful introduction to the technique and how it can be applied in medicine (Rigaud et al., 1996; Valentinuzzi, 1996). For the purposes of the current chapter, it is sufficient to recognize that the values of the real and imaginary components are dependent on the properties and structures of the underlying biological tissue. When this concept is applied to skin, we see that different elements in the skin give rise to different components of the overall impedance signal. For example, the sweat glands and hair follicles are predominantly resistive in nature, whilst the lipid laden regions of the dermis are dominantly capacitive in nature. The skin can therefore be modeled as a series of resistors and capacitors (Figure 7). Applying this model to skin, it is easy to see how the skin impedance is dependent on a whole host of anatomical and physiological variables within the skin structure. 


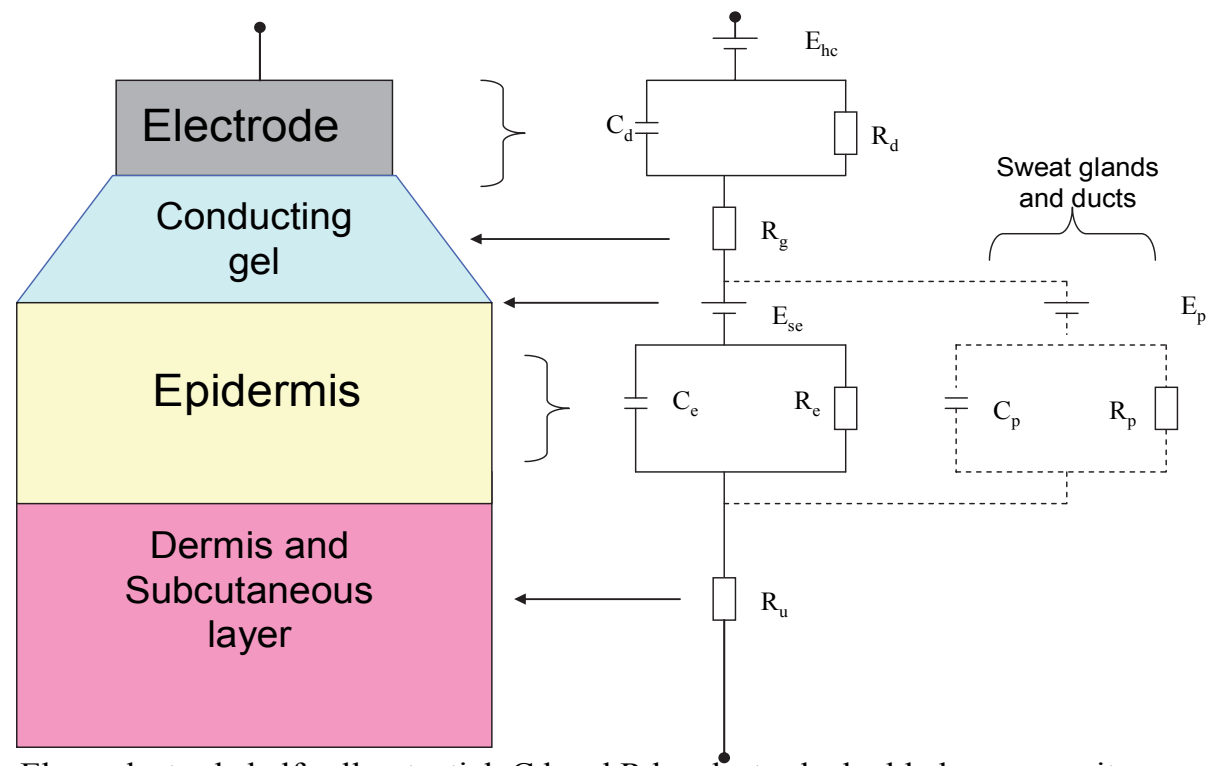

Ehc - electrode half cell potential, $\mathrm{Cd}$ and $\mathrm{Rd}$ - electrode double layer capacitance and resistance, $\mathrm{Rg}$ - gel resistance, Ese - potential due to ionic differences between gel and stratum corneum (SC), Ep - potential developed due to skin pores (release of ions in sweat), Ce and $\mathrm{Re}$ - capacitance and resistance of SC, $\mathrm{Cp}$ and $\mathrm{Re}$ - capacitance and resistance of skin pores, $\mathrm{Rg}$ - dermis and subcutaneous resistance.

Fig. 7. One representation of how skin can be modeled electrically.

Our specific interest has been in examining how skin impedance might be usefully deployed in the calibration of minimally invasive transdermal sensing applications. Briefly, we consider that the flux, F, of any given molecule across the skin will be a function of skin permeability, $\mathrm{P}$, such that,

$$
\mathrm{F}=\mathrm{C} \times \mathrm{P}+\mathrm{k}
$$

Where $\mathrm{C}$ is the molecule concentration in blood/interstitial fluid, and $\mathrm{k}$ is a constant to be determined.

Given the impracticality of measuring $\mathrm{P}$ in people, we have been investigating if various impedance derived parameters ( $Z$ values), taken from measurements of human skin in vivo can be used to provide an indication of the variation in $\mathrm{P}$ from person to person and day to day. Our results indicate that this approach may be able to provide individual permeability values that are parameter and person specific, thus removing the requirement for the time consuming and often invasive calibration methods which are limitations of all existing transdermal monitoring devices. In one recent study in diabetic patients completed in 2009, we have shown that the rate of transdermal potassium flux collected in the gel of iontophoresis electrodes placed on the skin of a diabetic patient can be directly related to a normalized impedance parameter, measured through the same 
electrodes. Figure 8 shows that the normalized impedance on patient skin can be correlated with transdermal flux. This opens the debate again on the possible uses of electrolytes as internal standards that can be used to calibrate iontophoresis systems for skin permeability and transdermal extractions.

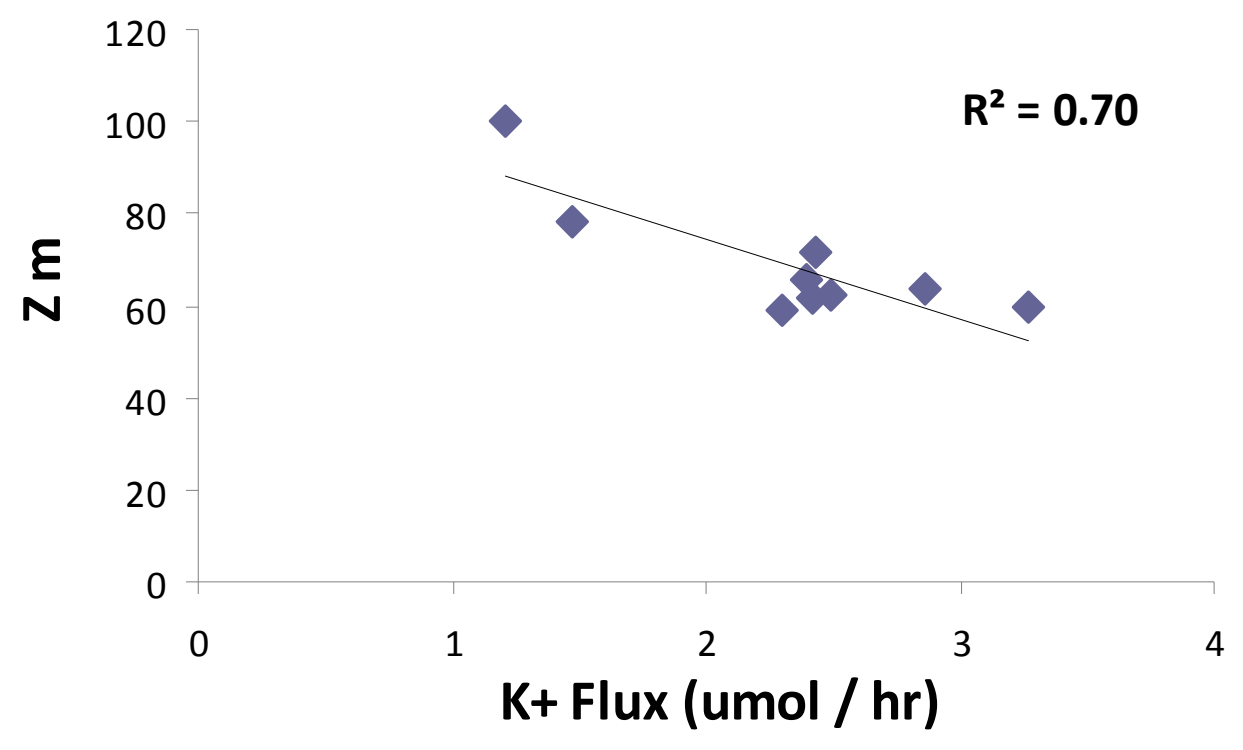

Fig. 8. Normalised impedance parameter, $\mathrm{Zm}$, versus transdermal potassium ion flux in a Type II Diabetes patient group $(n=9)$.

\section{Conclusion}

Demand for clinical diagnostics in the home and community will grow as disease states develop in an increasingly aged world population. As yet biosensors have not overcome the challenges that host responses to implantation generate for sensor systems and therefore long term implantation of sensors for diagnostic monitoring is not a reality. Minimally invasive monitoring via the transdermal route is open to further development for wearable or point of care systems and combining know how from drug delivery research and miniaturised sensors opens new possibilities in device design for clinical monitoring. A key aspect of successful deployment of devices in this field will be the creation of multidisciplinary working groups that cross the clinical interface and the resolution of issues around calibration of devices. 


\section{References}

Acierno, MJ, Johnson, ME, Eddleman, LA, Mitchell, MA (2008) Measuring statistical agreement between four point of care (POC) lactate meters and a laboratory blood analyzer in cats. Journal of Feline Medicine E Surgery 10 (2): 110-114

Arora, A, Prausnitz, MR, Mitragotri, S (2008) Micro-scale devices for transdermal drug delivery. Int J Pharm 364(2): 227-236.

Badugu, R, Lakowicz, JR, Geddes, CD (2005) Fluorescence sensors for monosaccharides based on the 6-methylquinolinium nucleus and boronic acid moiety: potential application to ophthalmic diagnostics. Talanta 65(3): 762-768.

Badugu R, Lakowicz JR, and Geddes CD (2003) A Glucose Sensing Contact Lens: A NonInvasive Technique for Continuous Physiological Glucose Monitoring. Journal of Fluorescence 13 (5)

Cancel, LM, Tarbell, JM, Ben-Jebria, A (2004) Fluorescein permeability and electrical resistance of human skin during low frequency ultrasound application. Journal of pharmacy and pharmacology 56: 1109-1118

Cevc, G, Vierl, U (2010) Nanotechnology and the transdermal route: A state of the art review and critical appraisal. Journal of Controlled Release 141(3): 277-299.

Ching, CTS, Buisson, Y, Connolly, P (2008a) The effect of pulsed bipolar dc on the simultaneous extraction of glucose and lactate by reverse iontophoresis. Sensors and Actuators B: Chemical 129(2): 504-509.

Ching, TS, Connolly, P (2008b) Simultaneous transdermal extraction of glucose and lactate from human subjects by reverse iontophoresis. Int J Nanomedicine 3(2): 211-223.

Chizmadzhev, YA, Indenbom, AV, Kuzmin, PI, Galichenko, SV, Weaver, JC, Potts, RO (1998) Electrical properties of skin at moderate voltages: contribution of appendageal macropores. Biophys J 74(2 Pt 1): 843-856.

Cornwell, PA, Barry, BW (1995) Effects of penetration enhancer treatment on the statistical distribution of human skin permeabilities. International Journal of Pharmaceutics 117(1): 101-112.

Cox, M (2009) An Overview of Continuous Glucose Monitoring Systems. Journal of Pediatric Health Care 23 (5): 344-347

Curdy, C, Kalia, YN, Guy, RH (2001) Non-invasive assessment of the effects of iontophoresis on human skin in-vivo. J Pharm Pharmacol 53(6): 769-777.

Curdy, C, Kalia, YN, Guy, RH (2002) Post-iontophoresis recovery of human skin impedance in vivo. European Journal of Pharmaceutics and Biopharmaceutics 53(1): 15-21.

Daniel, KD, Kim, GY, Vassiliou, CC, Galindo, M, Guimaraes, AR, Weissleder, R, Charest, A, Langer, R, Cima, MJ (2009) Implantable diagnostic device for cancer monitoring. Biosens Bioelectron 24(11): 3252-3257.

Delgado-Charro, MB, Guy, RH (1994) Characterization of convective solvent flow during iontophoresis. Pharm Res 11(7): 929-935.

Delgado-Charro, B, Rodriguez-Bayn AM, Richard H. Guy (2005) Iontophoresis of nafarelin: effects of current density and concentration on electrotransport in vitro. Journal of Controlled Release 35: $35-40$ 
Escobar-Chavez, JJ, Bonilla-Martinez, D, Villegas-Gonzalez, MA, Rodriguez-Cruz, IM, Dominguez-Delgado, CL (2009) The use of sonophoresis in the administration of drugs throughout the skin. J Pharm Pharm Sci 12(1): 88-115.

Ferrante do Amaral, CE, Wolf, B (2008) Current development in non-invasive glucose monitoring. Medical Engineering \& Physics 30(5): 541-549.

Gifford, R, Kehoe, JJ, Barnes, SL, Kornilayev, BA, Alterman, MA, Wilson, GS (2006) Protein interactions with subcutaneously implanted biosensors. Biomaterials 27(12): 25872598.

Girardin, CM, Huot, C, Gonthier, M, Delvin, E (2009) Continuous glucose monitoring: a review of biochemical perspectives and clinical use in type 1 diabetes. Clin Biochem 42(3): 136-142.

Howard, JP, Drake, TR, Kellogg, DL, Jr. (1995) Effects of alternating current iontophoresis on drug delivery. Arch Phys Med Rehabil 76(5): 463-466.

HSBC Global Research (2006) Medtronic, Diabetes: sizing the market for real time continuous glucose monitors. HSBC Research, 22 June 2006

http:/ / www.research.hsbc.com/midas/Res/RDV?p=pdf\&key=ate2b8rygn\&name $=127057 . \mathrm{PDF}$

Kissinger, PT (2005) Biosensors-a perspective. Biosens Bioelectron 20(12): 2512-2516.

Künzelmann, U, Böttcher, H (1997) Biosensor properties of glucose oxidase immobilized within $\mathrm{SiO} 2$ gels. Sensors and Actuators B: Chemical 39(1-3): 222-228.

Kost J, Mitragotri S, Gabbay RA, Pishko M, Langer R. Transdermal monitoring of glucose and other analytes using ultrasound. Nat Med 2000; 6: 347-350.

Kulcu, E, Tamada, JA, Reach, G, Potts, RO, Lesho, MJ (2003) Physiological differences between interstitial glucose and blood glucose measured in human subjects. Diabetes Care 26(8): 2405-2409.

Kurnik, RT, Berner, B, Tamada, J, Potts, RO (1998) Design and Simulation of a Reverse Iontophoretic Glucose Monitoring Device. Journal of The Electrochemical Society 145(12): 4119-4125.

Lademann, J, Jacobi, U, Surber, C, Weigmann, HJ, Fluhr, JW (2009) The tape stripping procedure--evaluation of some critical parameters. Eur J Pharm Biopharm 72(2): 317323.

Leboulanger, B, Guy, RH, Delgado-Charro, MB (2004) Non-invasive monitoring of phenytoin by reverse iontophoresis. Eur J Pharm Sci 22(5): 427-433.

Ledger, PW Skin biological issues in electrically enhanced transdermal delivery. Advanced Drug Delivery Reviews 9(2-3): 289-307.

Liberty, HJ, Johnson, BD, Fortner, N, Randolph, D (2003) Detecting crack and other cocaine use with fastpatches. Addict Biol 8(2): 191-200.

Liu, H, Li, H, Ying, T, Sun, K, Qin, Y, Qi, D (1998) Amperometric biosensor sensitive to glucose and lactose based on co-immobilization of ferrocene, glucose oxidase, [beta]-galactosidase and mutarotase in [beta]-cyclodextrin polymer. Analytica Chimica Acta 358(2): 137-144.

Manabe, E, Numajiri, S, Sugibayashi, K, Morimoto, Y (2000) Analysis of skin permeationenhancing mechanism of iontophoresis using hydrodynamic pore theory. Journal of Controlled Release 66 (2-3): 149-158 
Marbach, R, Koschinsky, Th, Gries, FA, Heise, HM (1993) Noninvasive Blood Glucose Assay by Near-Infrared Diffuse Reflectance Spectroscopy of the Human Inner Lip. Applied Spectroscopy 47 (7): 875-881

McColl, D (2001) A glucose biosensor for a hydrogel environment. University of Strathclyde, MSc Thesis

Meurin, Ph, Tabet, JY, Monchi, M, Weber, H, Fabre, C, Gibier, B, Renaud, N Ben Driss, A (2009) Accuracy of a portable international normalized ratio monitor for patients receiving a low molecular weight heparin as a bridge pending full oral anticoagulant efficacy. Thrombosis Research 125 (2): 192-195

Mitragotri, S, Coleman, M, Kost, J, Langer, R (2000) Transdermal extraction of analytes using low-frequency ultrasound. Pharm Res 17(4): 466-470

Mitragotri, S (2000) Synergistic effect of enhancers for transdermal drug delivery. Pharm Res 17(11): 1354-1359.

Moschou, EA, Sharma, BV, Deo, SK, Daunert, S (2004) Fluorescence Glucose Detection: Advances Toward the Ideal In vivo Biosensor. Journal of Fluorescence 14 (5): 53547

Mukerjee, EV, Collins, SD, Isseroff, RR, Smith, RL (2004) Microneedle array for transdermal biological fluid extraction and in situ analysis. Sensors and Actuators A: Physical 114(2-3): 267-275.

Newman, JD, Turner, AP (2005) Home blood glucose biosensors: a commercial perspective. Biosens Bioelectron 20(12): 2435-2453.

Numajiri, S, Sugibayashi, K, Morimoto, Y (1993) Non-invasive sampling of lactic acid ions by iontophoresis using chloride ion in the body as an internal standard. J Pharm Biomed Anal 11(10): 903-909.

N. S. Oliver, C. Toumazou, A. E. G. Cass and D. G. Johnston (2009) Glucose sensors: a review of current and emerging Technology, Diabetic Medicine, 26(3): 197-210

Pantelopoulos, A, Bourbakis, NG (2010) A Survey on Wearable Sensor-Based Systems for Health Monitoring and Prognosis. Systems, Man, and Cybernetics, Part C: Applications and Reviews, IEEE Transactions on 40(1): 1-12.

Pellett, MA, Hadgraft, J, Roberts, MS (1999) The back diffusion of glucose across human skin in vitro. International Journal of Pharmaceutics 193(1): 27-35.

Pickup, JC, Williams, G (1997) Textbook of Diabetes 2nd Edition. Blackwell Science Ltd, Oxford

Pickup, JC, Hussain, F, Evans, ND, Rolinski, OJ, Birch, DJ (2005) Fluorescence-based glucose sensors. Biosens Bioelectron 20(12): 2555-2565.

Posnett, J, Franks, PJ (2008) The burden of chronic wounds in the UK. Nurs Times 104(3): 4445.

Rigaud, B, Morucci, JP, Chauveau, N (1996) Bioelectrical impedance techniques in medicine. Part I: Bioimpedance measurement. Second section: impedance spectrometry. Crit Rev Biomed Eng 24(4-6): 257-351.

Riviere, JE, Heit, MC (1997) Electrically-assisted transdermal drug delivery. Pharm Res 14(6): 687-697.

Ruddy, SB, Hadzija, BW (1995) The role of stratum corneum in electrically facilitated transdermal drug delivery. I. Influence of hydration, tape-stripping and 
delipidization on the DC electrical properties of skin. Journal of Controlled Release 37(3): 225-238.

Saby, C, Mizutani, F, Yabuki, S (1995) Glucose sensor based on carbon paste electrode incorporating poly(ethylene glycol) -modified glucose oxidase and various mediators. Analytica Chimica Acta 304(1): 33-39.

Santi, P, Guy, RH (1996a) Reverse iontophoresis -- parameters determining electroosmotic flow. II. Electrode chamber formulation. Journal of Controlled Release 42(1): 29-36.

Santi, P, Guy, RH (1996b) Reverse iontophoresis -- Parameters determining electroosmotic flow: I. pH and ionic strength. Journal of Controlled Release 38(2-3): 159-165.

Sato, N, Okuma, H (2006) Amperometric simultaneous sensing system for d-glucose and 1lactate based on enzyme-modified bilayer electrodes. Analytica Chimica Acta 565(2): 250-254.

Schrader, W, Meuerb, P, Popp, J, Kiefer, W, Menzebach, JU, Schrader, B (2005) Non-invasive glucose determination in the human eye. Journal of Molecular Structure 735-736: 299-306

Sieg, A, Guy, RH, Delgado-Charro, MB (2004a) Noninvasive glucose monitoring by reverse iontophoresis in vivo: application of the internal standard concept. Clin Chem 50(8): 1383-1390.

Sieg, A, Guy, RH, Delgado-Charro, MB (2003) Reverse iontophoresis for noninvasive glucose monitoring: the internal standard concept. J Pharm Sci 92(11): 2295-2302.

Sieg, A, Guy, RH, Delgado-Charro, MB (2004b) Simultaneous extraction of urea and glucose by reverse iontophoresis in vivo. Pharm Res 21(10): 1805-1810.

Sieg, A, Jeanneret, F, Fathi, M, Hochstrasser, D, Rudaz, S, Veuthey, JL, Guy, RH, DelgadoCharro, MB (2009) Extraction of amino acids by reverse iontophoresis in vivo. Eur J Pharm Biopharm 72(1): 226-231.

Sintov, AC, Krymberk, I, Daniel, D, Hannan, T, Sohn, Ze, Levin, G (2003) Radiofrequencydriven skin microchanneling as a new way for electrically assisted transdermal delivery of hydrophilic drugs. Journal of Controlled Release 89(2): 311-320.

Tamada, JA, Bohannon, NJ, Potts, RO (1995) Measurement of glucose in diabetic subjects using noninvasive transdermal extraction. Nat Med 1(11): 1198-1201.

Tezel A, Sens A, Tuchscherer J, Mitragotri S (2001) Frequency Dependence of Sonophoresis. Pharmaceutical Research (18) 12: 1694-1700

Thong, HY, Zhai, H, Maibach, HI (2007) Percutaneous penetration enhancers: an overview. Skin Pharmacol Physiol 20(6): 272-282.

Tierney, MJ, Tamada, JA, Potts, RO, Jovanovic, L, Garg, S (2001) Clinical evaluation of the GlucoWatch biographer: a continual, non-invasive glucose monitor for patients with diabetes. Biosens Bioelectron 16(9-12): 621-629.

Tura, A, Maran, A, Pacini, G (2007) Non-invasive glucose monitoring: assessment of technologies and devices according to quantitative criteria. Diabetes Res Clin Pract 77(1): 16-40. 
Turner, APF, Karube, I, Wilson, GS, Worsfold, PJ (1987) Biosensors: fundamentals and applications : OUP, Oxford, 1987 (ISBN 0-19-854724-2). xvi+770 pp. Price £60.00. Analytica Chimica Acta 201: 363-364.

Ueda, H, Sugibayashi, K, Morimoto, Y (1995) Skin penetration-enhancing effect of drugs by phonophoresis. Journal of Controlled Release 37(3): 291-297.

Uemura, N, Harkey, MR, Nath, RP, Henderson, GL, Mendelson, JE, Jones, RT (2004) Delayed disposition of cocaine in sweat patches and in skin blister: cocaine may migrate from plasma to sweat patches via interstitial fluid. Clin Pharmacol Ther 75(2): P44-P44.

UK Prospective Diabetes Study Group (1998a) Effect of intensive blood-glucose control with metformin on complications in overweight patients with type 2 diabetes (UKPDS 34). The Lancet 352(9131): 854-865.

UK Prospective Diabetes Study Group (1998b) Efficacy of atenolol and captopril in reducing risk of macrovascular and microvascular complications in type 2 diabetes: UKPDS 39. BMJ 317(7160): 713-720.

UK Prospective Diabetes Study Group (1998c) Intensive blood-glucose control with sulphonylureas or insulin compared with conventional treatment and risk of complications in patients with type 2 diabetes (UKPDS 33). The Lancet 352(9131): 837-853.

UK Prospective Diabetes Study Group (1998d) Tight blood pressure control and risk of macrovascular and microvascular complications in type 2 diabetes: UKPDS 38. BMJ 317(7160): 703-713.

U.S. Food and Drugs Adminstration, Summary of safety and effectiveness, in Pre Market Approval, P990026, 01 April 2011, http://www.accessdata.fda.gov/cdrh_docs/pdf/P990026b.pdf

U.S. Food and Drugs Adminstration, Summary of safety and effectiveness, in Pre Market Approval, P990026S008b, 01 April 2011, http://www.accessdata.fda.gov/cdrh_docs/pdf/P990026S008b.pdf

Vaddiraju, S, Tomazos, I, Burgess, DJ, Jain, FC, Papadimitrakopoulos, F (2010) Emerging synergy between nanotechnology and implantable biosensors: a review. Biosens Bioelectron 25(7): 1553-1565.

Valentinuzzi, ME (1996) Bioelectrical impedance techniques in medicine. Part I: Bioimpedance measurement. First section: general concepts. Crit Rev Biomed Eng 24(4-6): 223-255.

Vanbever, R, Preat, VV (1999) In vivo efficacy and safety of skin electroporation. Adv Drug Deliv Rev 35(1): 77-88.

Walsh, JM, Terdiman, JP (2003) Colorectal cancer screening: scientific review. JAMA 289(10): 1288-1296.

Watt, BE, Proudfoot, AT, Vale, JA (2004) Hydrogen peroxide poisoning. Toxicol Rev 23(1): 51-57.

Wesselingh, JA (1993) Controlling diffusion. Journal of Controlled Release 24(1-3): 47-60.

Westrin, BA, Axelsson, A, Zacchi, G (1994) Diffusion measurement in gels. Journal of Controlled Release 30(3): 189-199. 
Wickramasinghe, Y, Yang, Y, Spencer, SA (2004) Current problems and potential techniques in in vivo glucose monitoring. J Fluoresc 14(5): 513-520.

WHO, 2004. Diabetes Action Now: an Initiative of the World Health Organisation and the International Diabetes Federation. WHO, Geneva, Switzerland.

WHO, 2006. The Global Burden of Disease 2004-Update. WHO, Geneva, Switzerland.Lima.

WHO, 2008, The World Health Report 2008 - primary Health Care (Now More Than Ever). WHO, Geneva, Switzerland. 


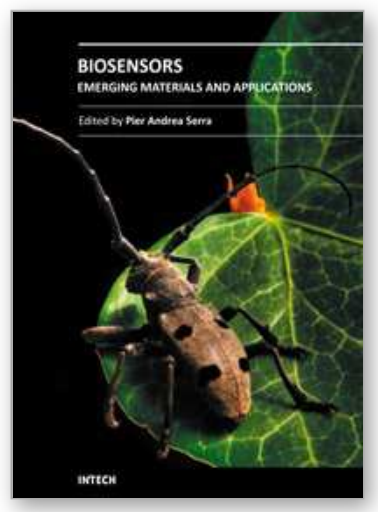

\author{
Biosensors - Emerging Materials and Applications \\ Edited by Prof. Pier Andrea Serra
}

ISBN 978-953-307-328-6

Hard cover, 630 pages

Publisher InTech

Published online 18, July, 2011

Published in print edition July, 2011

\begin{abstract}
A biosensor is a detecting device that combines a transducer with a biologically sensitive and selective component. Biosensors can measure compounds present in the environment, chemical processes, food and human body at low cost if compared with traditional analytical techniques. This book covers a wide range of aspects and issues related to biosensor technology, bringing together researchers from 19 different countries. The book consists of 27 chapters written by 106 authors and divided in three sections: Biosensors Technology and Materials, Biosensors for Health and Biosensors for Environment and Biosecurity.
\end{abstract}

\title{
How to reference
}

In order to correctly reference this scholarly work, feel free to copy and paste the following:

Christopher McCormick, David Heath and Patricia Connolly (2011). Minimally Invasive Sensing, Biosensors Emerging Materials and Applications, Prof. Pier Andrea Serra (Ed.), ISBN: 978-953-307-328-6, InTech, Available from: http://www.intechopen.com/books/biosensors-emerging-materials-and-applications/minimallyinvasive-sensing

\section{INTECH}

open science | open minds

\section{InTech Europe}

University Campus STeP Ri

Slavka Krautzeka 83/A

51000 Rijeka, Croatia

Phone: +385 (51) 770447

Fax: +385 (51) 686166

www.intechopen.com

\section{InTech China}

Unit 405, Office Block, Hotel Equatorial Shanghai

No.65, Yan An Road (West), Shanghai, 200040, China

中国上海市延安西路65号上海国际贵都大饭店办公楼 405 单元

Phone: +86-21-62489820

Fax: +86-21-62489821 
(C) 2011 The Author(s). Licensee IntechOpen. This chapter is distributed under the terms of the Creative Commons Attribution-NonCommercialShareAlike-3.0 License, which permits use, distribution and reproduction for non-commercial purposes, provided the original is properly cited and derivative works building on this content are distributed under the same license. 